at $50 \mathrm{mM}$ with the spectrometer set at a microwave frequency of $9.77 \mathrm{GHz}$ with $20 \mathrm{~mW}$ of microwave power and a modulation amplitude of $0.5 \mathrm{G}$. Treatment with Cu-Zn SOD (Sigma), catalase (Sigma), DP (Toronto Research), or N-acetyl-L-cysteine (Sigma) was for 20 min before recording of EPR spectra. Treatment with the FPTase inhibitor H-AmpambPhe-Met-OH (LC Laboratories) was for 48 hours before obtaining spectra. At this concentration and with this duration of treatment, no cellular toxicity was observed.

23. The empty expression plasmid pEXV and the expression plasmids pEXVracV12 (encoding myc epitope-tagged constitutively active Rac1), pEXVracN17 (dominant negative Rac1), pCMVrasN17 (dominant negative Ras), and pS3CAT (catalase) have been described $(15,21)$. Transient transfections were done according to the manufacturer's recommendations using $15 \mu \mathrm{l}$ of Lipofectamine
(Gibco) and $5 \mu \mathrm{g}$ of DNA per $10^{5}$ cells. EPR spectra were obtained 48 hours after transfection. For the LUCL assay, $1 \times 10^{5}$ transfected cells were replated onto each tissue culture insert 24 hours after transfection and the assay was performed 24 hours later. Protein immunoblot analysis was done on cells 48 hours after transfection.

24. Established A6, Neo, and Raf-transformed cell lines $(10,11)$ were maintained in Dulbecco's modified Eagle's medium with $10 \%$ fetal bovine serum (Gibco) and G418 (250 $\mu \mathrm{g} / \mathrm{ml}$, Sigma). Expression of $\mathrm{H}$-Ras ${ }^{\mathrm{V} 12}$ in $\mathrm{A} 6$ cells was confirmed by protein immunoblotting with an antibody to $\mathrm{H}$-Ras (Oncogene Science). Serum starvation was carried out in $0.1 \%$ serum for 48 hours.

25. For immunoprecipitation and protein immunoblotting, cells were harvested in complete lysis buffer [145 mM NaCl, $0.1 \mathrm{mM} \mathrm{MgCl}_{2}, 15 \mathrm{mM}$ Hepes, 10 mM EGTA (pH 7.0), 0.1\% Triton X-100, $20 \mu \mathrm{g} / \mathrm{m}$ each of chymostatin, pepstatin, antipain, and leupeptin, $1 \mathrm{mM}$ 4-(2-aminoethyl)benzylsulfonyl fluoride, and $1 \mathrm{mM} \mathrm{Na}$ orthovanadate]. Immunoprecipitates or total cell lysates were subjected to SDSpolyacrylamide gel electrophoresis (PAGE) and transferred to nitrocellulose filters. Blots were then probed with the indicated antibody and developed by ECL (Amersham).

26. M. Hibi et al., Genes Dev. 7, 2135 (1993).

27. We thank A. Hall for providing the plasmids encoding the Rac1 mutant isoforms, L. Feig for Ras ${ }^{N 17}$ CDNA, S. Erzurum for human catalase CDNA, and C. Lowenstein, C. Dang, and A. Hoang for helpful criticisms. Supported by NIH grant HL52315 (P.J.G.-C. and J.L.Z.), an Established Investigator Award, American Heart Association (P.J.G.-C.), and the Bernard Foundation.

8 November 1996; accepted 29 January 1997

\title{
A Member of the Frizzled Protein Family Mediating Axis Induction by Wnt-5A
}

\author{
Xi He, ${ }^{\star} \dagger$ Jean-Pierre Saint-Jeannet, Yanshu Wang, \\ Jeremy Nathans, Igor Dawid, Harold Varmus
}

In Xenopus laevis embryos, the Wingless/Wnt-1 subclass of Wnt molecules induces axis duplication, whereas the Wnt-5A subclass does not. This difference could be explained by distinct signal transduction pathways or by a lack of one or more Wnt-5A receptors during axis formation. Wnt-5A induced axis duplication and an ectopic Spemann organizer in the presence of $\mathrm{hFz5}$, a member of the Frizzled family of seven-transmembrane receptors. Wnt-5A/hFz 5 signaling was antagonized by glycogen synthase kinase- 3 and by the amino-terminal ectodomain of $\mathrm{hFz} 5$. These results identify $\mathrm{hFz} 5$ as a receptor for Wnt-5A.

The Drosophila melanogaster tissue polarity gene frizzled $(f z)$, which encodes a receptortype protein $(\mathrm{Fz})$ with seven putative transmembrane helixes (1), belongs to a family of related genes found in Caenorhabditis elegans, Drosophila, zebrafish, chicken, and mammals (2-4). DFz2, a Fz-type protein from Drosophila, functions as a receptor for the Wingless (Wg) protein (4), a member of the Wnt family of secreted signaling molecules essential for animal development (5). Upon transfection of Drosophila tissue culture cells, DFz2 confers both the ability to bind Wg at the cell surface and Wg responsiveness, as measured by accumulation of Armadillo protein (4). Certain mammalian $\mathrm{Fz}$ proteins also confer the $\mathrm{Wg}$

$\mathrm{X} . \mathrm{He}$ and $\mathrm{H}$. Varmus, National Cancer Institute, Building 49, Room 4A56, National Institutes of Health, Bethesda, MD 20892, USA.

J.-P. Saint-Jeannet and I. Dawid, National Institute of Child Health and Human Development, National Institutes of Health, Bethesda, MD 20892, USA.

Y. Wang and J. Nathans, Howard Hughes Medical Institute, Department of Molecular Biology and Genetics, The Johns Hopkins University School of Medicine, Baltimore, MD 21205, USA

*Present address: Division of Neuroscience, Enders 379 Children's Hospital/Harvard Medical School, 300 Longwood Avenue, Boston, MA 02115, USA

†To whom correspondence should be addressed. Email: xhe@nhgri.nih.gov binding property to transfected cells (4) and can enhance the localization of Wnt-8 to the plasma membrane in Xenopus laevis embryos (6). Thus, the $\mathrm{fz}$ gene family may encode receptors for Wnt molecules, but the scarcity of soluble Wnt proteins complicates the study of ligand-receptor interactions and their specificity. Therefore, we used the Xenopus embryo to assay for such interactions and their specificity.

Mouse Wnt-1, Xenopus wnt-3A (Xwnt3A), Xwnt-8, and Drosophila Wg induce dorsal axis duplication when small amounts, usually 1 to $10 \mathrm{pg}$, of their corresponding RNAs are injected into the ventral side of early Xenopus embryos (7). In contrast, Xwnt-5A RNA fails to do so even after ventral injection at higher doses ( 75 pg to 1 ng per embryo); instead, dorsal injection of Xwnt-5A RNA generates head and tail defects that may result from perturbation of cell movements during gastrulation (8). Xwnt-4 and Xwnt-11 behave similarly to Xwnt-5A (9). The Xwnt-8 dorsalizing function is observed before the mid-blastula transition when zygotic transcription begins, whereas the Xwnt-5A effect occurs after mid-blastula transition $(7,8,10,11)$. The difference between the effects of Xwnt- 8 and Xwnt-5A may reflect the acti- vation of distinct signaling pathways (11) or the lack of one or more functional Xwnt$5 \mathrm{~A}$ receptors during axis formation.

To examine whether a particular Fz protein can function as an Xwnt-5A receptor, synthetic RNAs corresponding to Dfz2 (4) and six mammalian $\mathrm{fz} c \mathrm{cNAs}-\mathrm{mfz} 3, \mathrm{mfz}^{4}$, $\mathrm{mfz} 6, \mathrm{mfz} 7, \mathrm{mfz} 8$ (from mouse), and hfz5 [from human (3)] -were pooled into two groups and coinjected with 10 pg of Xwnt$5 \mathrm{~A}$ RNA into the ventral side of embryos at the four-cell stage (12). Injection of Xwnt$5 \mathrm{~A}$ alone, either $\mathrm{fz}$ group alone, or Xwnt$5 \mathrm{~A}$ together with $\mathrm{fz}$ group $2(\mathrm{mfz} 3, \mathrm{mfz} 4$, $\mathrm{mfz} 6$, and $\mathrm{mfz} 7$ ) produced no phenotypic effects (Fig. 1A). However, coinjection of Xwnt-5A with $\mathrm{fz}$ group 1 RNAs (Dfz2, hfz5, and $\mathrm{mfz} 8$ ) induced extensive dorsal axis duplication (Fig. 1A) (13); in many cases, duplication was complete, as determined by the presence of anterior structures such as the eyes and the cement gland. When the three $\mathrm{fz}$ RNAs in group 1 were individually tested, Xwnt-5A plus hfz5 generated similar degrees of axis duplication, whereas Xwnt$5 \mathrm{~A}$ plus $\mathrm{Dfz} 2$ or $\mathrm{mfz} 8$ did not (Fig. 1B). Thus, $\mathrm{hFz} 5$ alone among the $\mathrm{Fz}$ proteins tested is responsible for mediating axis induction by Xwnt-5A. Dorsal injection of the same concentration of Xwnt-5A plus hfz5 RNAs produced no axis duplication and injected embryos appeared normal.

The mature Wnt-5A proteins (after cleavage of the signal peptides) are 100\% identical between mouse and human, and 95\% identical between mouse and Xenopus $(8,14)$. Given this high degree of sequence identity, it is not surprising that murine wnt-5A RNA (15) also induced axis duplication when coinjected with hfz5 RNA, albeit less efficiently (Fig. 1C). The lower efficiency might be due to effects of untranslated regions in the murine wnt-5A construct (15) on RNA stability or translation efficiency or both.

Axis duplication by Xwnt-5A plus $f z$ group 1 or $\mathrm{hfz} 5$, as described above, was observed in 14 of 20 embryo batches tested 
during the course of this study. In these cases, 90 to $100 \%$ of the injected embryos showed axis duplication, of which 17 to $82 \%$ included eyes (16). In the remaining 6 of 20 embryo batches, the same coinjection induced no or few axis duplications $(<30 \%$, none complete). The reason for this poor response in some batches is unknown. Possible explanations include variations in the stability of injected RNAs or translated proteins, or both, the efficiency of Wnt-5A secretion, the assembly or localization of $\mathrm{hFz} 5$ protein, or both, and the availability of unknown coreceptor molecules.

It should be noted that whereas $\mathrm{hFz} 5$ is more closely related to $\mathrm{DFz}_{2}$ and $\mathrm{mFz} 8$ than to any other known $\mathrm{Fz}$ proteins $(3,4)$, neither $\mathrm{DFz}_{2}$ nor $\mathrm{mFz} 8$ cooperated with Xwnt-5A in axis induction. However, $\mathrm{DFz}_{2}$ appeared to be functional in Xenopus embryos, because the same concentration of Dfz2 RNA as used for coinjection with Xwnt-5A substantially enhanced axis induction by suboptimal amounts of wg RNA (17). The failure of the other $\mathrm{Fz}$ proteins to mediate Xwnt-5A function could be due to an inability to bind Xwnt-5A or an inability to signal in this context.

A dose-response curve illustrated that, in the presence of $0.4 \mathrm{ng}$ of $\mathrm{hf} 5 \mathrm{RNA}$ per embryo, 1 pg of Xwnt-5A RNA induced partial secondary axes whereas $20 \mathrm{pg}$ of Xwnt-5A RNA sometimes hyperdorsalized embryos (Fig. 2A). Although the relative protein levels have not been determined, the dose of Xwnt-5A RNA required for axis induction was in a similar range to effective doses of Xwnt-3A, Xwnt-8, wnt-1, and wg RNAs (7). Together, these data suggest a high degree of specificity of the interaction between Xwnt-5A and hFz5.

Embryos ventralized by ultraviolet (UV) irradiation (18) can be rescued by the $\mathrm{Wg} / \mathrm{Wnt}-1$ subclass of Wnt molecules (7). Xwnt-5A plus $\mathrm{hFz} 5$ could also restore dorsal development in UV-ventralized embryos, whereas neither Xwnt-5A nor hFz5 alone did (Fig. 2B). Many embryos rescued by Xwnt-5A plus $\mathrm{hFz} 5$ had a dorsoanterior index (DAI) (19) of 5, signifying normal development.

Histological examination (20) of embryos with duplicated axes revealed that Xwnt-5A plus hFz5 RNAs induced a full array of dorsal tissues, including notochord, neural tube, and somites (Fig. 3B). There is one notable difference between axes induced by Xwnt- 8 and those induced by Xwnt-5A plus hFz5: whereas the ectopic axes induced by Xwnt- 8 are often indistinguishable from the endogenous ones, the axes induced by Xwnt-5A and $\mathrm{hFz}_{2} 5$ are shorter in most cases, even when eyes and the cement gland are present (Fig. 3.A). This might reflect the previously
Fig. 1. Dorsal axis induction by Xwnt-5A mediated via $h F z 5 ; n$ represents the total number of embryos scored from two to six independent experiments; each bar represents the average percentage of axis duplication; the solid portion within each bar represents the average percentage of extensive axis duplication, which is defined by the presence of the cement gland and at least one eye in the duplicated axis. Unless otherwise specified, $10 \mathrm{pg}$ of wnt RNA or $400 \mathrm{pg}$ of each $\mathrm{fz} R \mathrm{RNA}$, or both, were injected per embryo. (A) Ventral injection of Xwnt-5A RNA together with RNA for $\mathrm{fz}$ group 1 (Dfz2, hfz5, and $\mathrm{mfz} 8$ ) induced axis duplication. Xwnt-5A RNA alone, $f z$ group 1 or group 2 ( $m f z 3, m f z 4, m f z 6$, and mfz7) alone, and Xwnt-5A RNA plus $f z$ group 2 did not. (B) Xwnt-5A RNA induced axis duplication in the presence of $\mathrm{hfz} 5$ RNA, but not with Dfz2 or mfz8 RNA. (C) Murine wnt-5A RNA plus hFz5 also induced axis duplication.

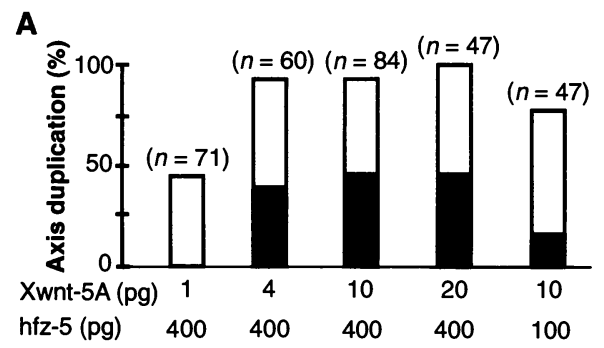

B

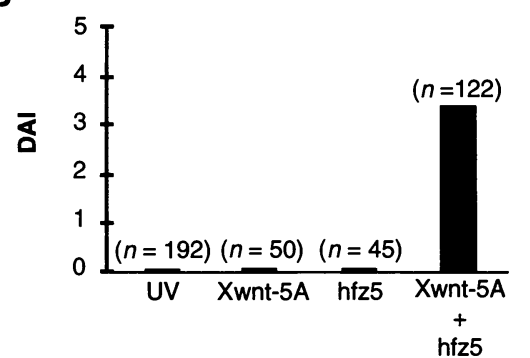

Fig. 2. (A) Dose-response curve of $X w n t-5 A$ and hfz5 RNAs for axis duplication. The bar legend is as in Fig. 1. (B) Xwnt-5A plus hFz5 rescued UV ventralized embryos. All embryos were UV-irradiated and were either left uninjected (UV), or injected with RNA for Xwnt-5A or hfz 5 alone, or a com bination of both (Xwnt-5A + hfz5). The DAl is defined in (19), with a DAl of 0 representing complete ventralization and a DAI of 5 equivalent to normal embryos. The average DAl from two to four experiments was shown.

described ability of Xwnt-5A to inhibit cell movements during gastrulation (8).

We tested whether Xwnt-5A plus hFz5 induce an ectopic Spemann organizer, as does Xwnt- 8 or Wnt-1, by examining the expression of the organizer-specific gene goosecoid (gsc) (21). Embryos injected with Xwnt-5A or hfz5 RNA alone expressed gsc only dorsally, as did uninjected controls; in
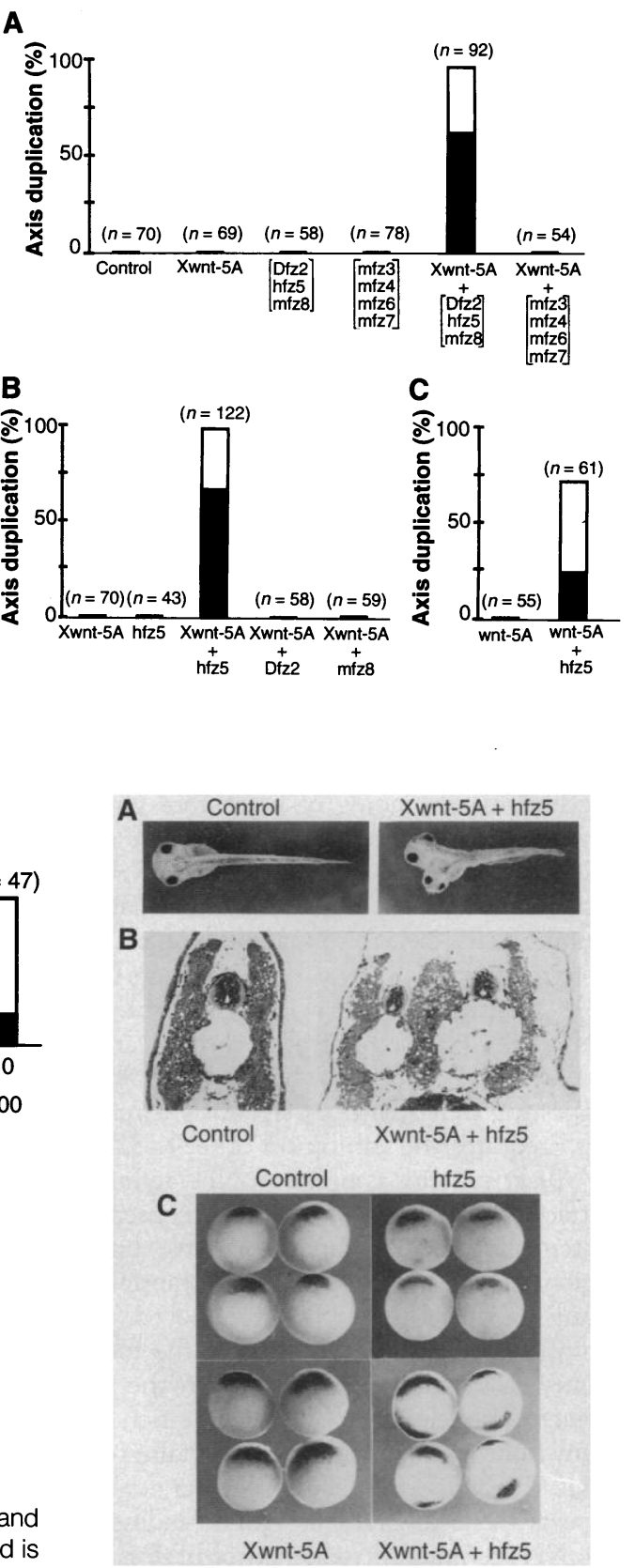

Fig. 3. Induction of anteriormost structures, dorsal axial tissues, and the Spemann organizer by Xwnt-5A plus hfz5. (A) Xwnt-5A plus hfz5 induced, axis duplication. An example of complete axis duplication with eyes in both axes is shown (stage 41). (B) Xwnt-5A plus hfz5 induced a complete set of dorsal tissues. Cross section in the trunk region of an embryo at stage 41 reveals the presence of a neural tube, notochord, and somites in both axes. (C) Xwnt-5A plus hfz5 induced ectopic gsc expression in embryos at stage 10.5 , as visualized by whole mount in situ hybridization.

contrast, embryos coinjected with Xwnt-5A and hfz5 RNAs exhibited two opposing domains of gsc expression, indicating the formation of an ectopic organizer (Fig. 3C)

Drosophila Wg function is mediated by inhibition of the zeste-white 3 (shaggy) gene 
Fig. 4. GSK-3ß and the secreted $\mathrm{NH}_{2}$-terminal ectodomain of hFz5 ( $h F z 5 N$ ) antagonize axis induction by Xwnt-5A plus hfz5. The bar legend for $(A)$ and $(B)$ is as in Fig. 1. (A) GSK-3 $\beta$ suppressed axis duplication. RNA for human GSK-3 $\beta$ was injected at $1 \mathrm{ng}$ per

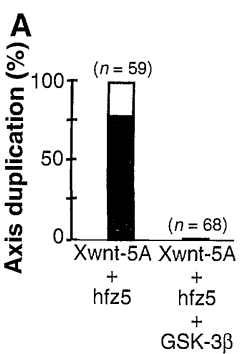
B尺 embryo. (B) hFz5N sup pressed axis duplication by Xwnt-5A plus hfz 5 in a dose-dependent manner. Dorsal injection of the same doses of hfz5N RNA did not affect the endogenous dorsal development. At higher doses (0.8 to $2 \mathrm{ng}$ RNA per embryo) some embryos with gastrulation defects were observed. (C) hFz5N inhibited Xwnt-5A/hFz5 signaling in neighboring cells. hfz5N RNA and RNAs for Xwnt-5A plus hfz 5 were injected into two separate neighboring blastomeres at the four-cell stage. This experiment was done in UV-ventralized embryos because of the semi-quantitative nature of the DAl score. Embryos were either left uninjected (UV) or injected with the following RNA combinations: I, Xwnt-5A (10 pg) plus hFz5 (400 pg) in one blastomere; II and III, Xwnt-5A plus hFz5 in one blastomere, then with 400 pg (II) or 800 pg (III) of hfz5N RNA in a neighboring blastomere; or IV, Xwnt-5A, hfz5, and hfz5N (800 pg of RNA) were coinjected into a single blastomere. The average DAl from three independent experiments is shown. Note that the overall inhibitory effect of hFz5N via coinjection (IV) and the separate injection (III) are similar.

product, the homolog of vertebrate glycogen synthase kinase-3 (GSK-3) (22), and the dorsalizing function of Xwnt-8 RNA in Xenopus is mimicked by dominant-negative mutant forms of GSK-3 and antagonized by overexpression of wild-type GSK-3 (23, 24). Coinjection of wild-type GSK-3 $\beta$ RNA (24) blocked dorsal axis duplication by Xwnt-5A plus hfz5 RNAs (Fig. 4A), suggesting that Xwnt- $5 \mathrm{~A}$ signaling through $\mathrm{hFz} 5$ requires the inhibition of GSK-3.

All Fz proteins contain an $\mathrm{NH}_{2}$-terminal extracellular domain composed of a conserved cysteine-rich domain and a variable linker region before the first putative transmembrane helix $(1,3)$. The $\mathrm{NH}_{2}$-terminal ectodomain of DFz2, when anchored to the membrane, promoted binding of $\mathrm{Wg}$ to the cell surface, and deletion of the cysteine-rich domain from $\mathrm{mFz}_{4}$ abolished Wg binding (4). Thus, the $\mathrm{NH}_{2}$-terminal extracellular domain appears to be involved in ligand binding. If the $\mathrm{NH}_{2}$-terminal ectodomain of $\mathrm{hF} z 5$ is involved in binding Xwnt-5A, its overexpression as a secreted molecule might prevent Xwnt-5A from binding to and signaling through $\mathrm{hFz} 5$. Indeed, coinjection of RNA coding for the $\mathrm{hFz} 5 \mathrm{NH}_{2}$-terminus ( $\mathrm{hF} z 5 \mathrm{~N}$ ), without provision of a membrane anchor (25), antagonized axis induction by Xwnt-5A plus $\mathrm{hFz} 5$ in a dose-dependent manner (Fig. 4B). Dorsal injection of $\mathrm{hf} z 5 \mathrm{~N}$ RNA alone did not affect endogenous dorsal axis formation (although gastrulation defects were seen in some embryos); the injected embryos appeared to have an intact neural groove at the neurula stage, and the anteriormost structures (eyes and the cement gland) were present at the tadpole stage (17). These results rule out the possibility that the effect of $\mathrm{hFz} 5 \mathrm{~N}$ in blocking axis duplication by Xwnt- $5 \mathrm{~A}$ plus $\mathrm{hFz} 5$ is due to nonspecific toxicity on dorsal development.

To further eliminate the possibility that the expression of $h F z 5 \mathrm{~N}$ causes a generalized defect in protein secretion, thus preventing Xwnt-5A or $\mathrm{hFz} 5$ or both from reaching the cell surface, we injected hf $55 \mathrm{~N}$ RNA and RNAs for Xwnt-5A plus hfz 5 into separate neighboring blastomeres. Under these conditions, $\mathrm{hF} z 5 \mathrm{~N}$ inhibited axial development induced by Xwnt-5A plus $\mathrm{hFz} 5$ to an extent similar to that observed after coinjection of a single blastomere (Fig. $4 \mathrm{C})$. This result demonstrates that secreted $\mathrm{hFz} 5 \mathrm{~N}$ can diffuse to neighboring cells to inhibit Xwnt-5A/hFz5 signaling.

In summary, our data indicate that in the presence of $\mathrm{hFz} 5$, Wnt-5A can transduce a signal similar to that of Xwnt-8 or Wnt-1, mediated by a pathway involving down-regulation of GSK-3. The simplest interpretation of these results is that $\mathrm{hF} z 5$ functions as a receptor for Wnt-5A. Our experiments also provide a general assay to address the relationships among 14 Wnt and eight Fz proteins thus far identified in mammals.

\section{REFERENCES AND NOTES}

1. C. R. Vinson, S. Conover, P. N. Adler, Nature 338 , 263 (1989); W.-J. Park, J. C. Liu, P. N. Adler, Mech. Dev. 45, 127 (1995).

2. S. D. Chan et al., J. Biol. Chem. 267, 25202 (1992); Z.-Y. Zhao, C. C. Lee, A. Baldini, C. T. Caskey, Genomics 27, 370 (1995): H. Sawa, L. Lobel, H. R. Horvitz, Genes Dev. 10, 2189 (1996).

3. Y. Wang et al., J. Biol. Chem. 271, 4468 (1996).

4. P. Bhanot et al., Nature 382, 225 (1996).

5. R. Nusse and H. E. Varmus, Cell 69, 1073 (1992); A. P. McMahon, Trends Genet. 8, 236 (1992).

6. J. Yang-Snyder, J. R. Miller, J. D. Brown, C.-J. Lai, R. T. Moon, Curr. Biol. 6, 1302 (1996).

7. A. P. McMahon and R. T. Moon, Cell 58, 1075 (1989); W. C. Smith and R. M. Harland, ibid. 67, 753 (1991); S. Sokol, J. L. Christian, R. T. Moon, D. A. Melton, ibid., p. 741; A. Chakrabarti, G. Matthews, A. Colman, L. Dale, Development 115, 355 (1992); S. L.
Wolda, C. J. Moody, R. T. Moon, Dev. Biol. 155, 46 (1993).

8. R. T. Moon et al., Development 119, 97 (1993).

9. S. S. Du, J. Purcell, J. L. Christian, L. McGrew, R. T. Moon, Mol. Cell. Biol. 15, 2625 (1995).

10. J. L. Christian and R. T. Moon, Genes Dev. 7, 13 (1993).

11. R. T. Moon et al., Development (Suppl.) (1993), p. 85.

12. All $f z c D N A s$ were subcloned in the pRK5 vector [C. Gorman, D. R. Gies, G. McCray, DNA Prot. Eng. Techn. 2, 3 (1990)] with an optimal Kozak consensus sequence for translation at the initiator ATG (CCACCATG, preceded by different restriction sites for subcloning) and with different lengths of $3^{\prime}$ untranslated regions. The details of each construct are available upon request Xwnt-5A was in $\mathrm{pSP} 64$ vector as described (8). All RNAs for injection were synthesized as capped transcripts in vitro with SP6 RNA polymerase (Ambion Megascript). Unless otherwise specified, RNAs were injected into the two cells nea the equatorial midline region at the four-cell stage. Embryo preparation and staging were performed as described (24).

13. Percentages of axis duplication were obtained by dividing the total number of embryos with a duplicated axis by the total number of embryos, scored from two to six independent experiments. Extensive axis duplication is defined by the presence of the cement gland and at least one eye in the duplicated axis.

14. B. J. Gavin, J. A. McMahon, A. P. McMahon, Genes Dev. 4, 2319 (1990); C. C. Clark et al., Genomics 18 $249(1993)$

15. Murine wnt-5 cDNA (14) was cloned in pCS2 + vector [D. L. Turner and H. Weintraub, Genes Dev. 8 , 1434 (1994)] as the Eco Rl and Xbal fragment, which contains about $60 \mathrm{bp} 5^{\prime}$ and $360 \mathrm{bp} 3^{\prime}$ untranslated regions.

16. Some variability exists in the completeness of duplicated axes or axial rescue in UV-treated embryos among different batches of embryos. One possibility is that the coinjection of Xwnt-5A and hfz5 RNAs inevitably causes more variable results than does injection of a single RNA species, because the two proteins derived from the injected RNAs must be translated, processed, and localized properly to function together. In injection of a single RNA, such as one for Xwnt- 8 , the variables are only associated with the Xwnt- 8 protein, because one or more receptors are provided endogenously. Another possibility is that Xwnt-5A inhibits gastrulation movements in gastrula stages (8); this later function of Xwnt-5A might affect the completeness of the axis to a variable degree.

17. X. He et al. data not shown.

18. J. C. Gerhart et al., Development (Suppl.) 107, 37 (1989).

19. K. R. Kao and R. P. Elinson, Dev. Biol. 127, 64 (1988).

20. Embryo preparation, staging, UV irradiation, fixation and sectioning, and whole-mount in situ hybridization were performed as described (24).

21. K. W. Y. Cho, B. Blumberg, H. Steinbeisser, E. M. DeRobertis, Cell 67, 1111 (1991).

22. E. Siegfried, T.-B. Chou, N. Perrimon, ibid. 71, 1167 (1992).

23. S. B. Pierce and D. Kimelman, Development 121, 755 (1995); I. Dominguez, K. Itoh, S. Y. Sokol, Proc Natl. Acad. Sci. U.S.A. 92, 8498 (1995).

24. X. He, J.-P. Saint-Jeannet, J. R. Woodgett, H. E. Varmus, I. B. Dawid, Nature 374, 617 (1995).

25. hfz5N was generated by introduction of a stop codon just before the first putative transmembrane helix (changing the preceding amino acids 237 and 238 from phenylalanine and tryptophan to threonine and arginine, respectively). The corresponding DNA fragment was subcloned in pCS2t.

26. The authors are grateful to R. Moon for Xwnt-5A plasmid. We thank B. Huang for technical help and B. Williams, J. Debnath, P. Schwartzberg, and M. Rebagliati for discussion and comments on the manuscript. X.H. is supported by the U.S. Army Breast Cancer Research Program. J.N. is an investigator of the Howard Hughes Medical Institute.

9 October 1996; accepted 7 January 1997 